Extended Maxwell-Garnett-Mie formulation applied to size dispersion of metallic nanoparticles embedded in host liquid matrix

Y. Battie' , A. Resano-Garcia, N. Chaoui, Y. Zhang, and A. En Naciri

Citation: The Journal of Chemical Physics 140, 044705 (2014); doi: 10.1063/1.4862995

View online: http://dx.doi.org/10.1063/1.4862995

View Table of Contents: http://aip.scitation.org/toc/jcp/140/4

Published by the American Institute of Physics

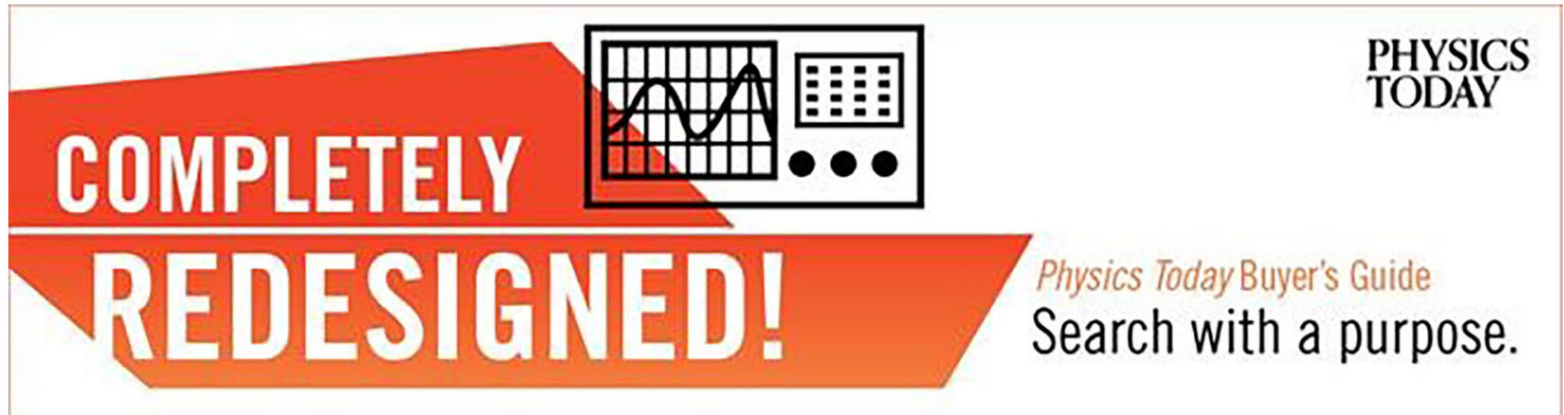




\title{
Extended Maxwell-Garnett-Mie formulation applied to size dispersion of metallic nanoparticles embedded in host liquid matrix
}

\author{
Y. Battie, ${ }^{1, a)}$ A. Resano-Garcia, ${ }^{1}$ N. Chaoui, ${ }^{1}$ Y. Zhang, ${ }^{2}$ and A. En Naciri ${ }^{1}$ \\ ${ }^{1}$ LCP-A2MC, Institut Jean Barriol, Université de Lorraine, 1 Bd Arago, 57070 Metz, France \\ ${ }^{2}$ LEM3, Université de Lorraine, Ile du Saulcy, $57045 \mathrm{Metz}$, France
}

(Received 23 September 2013; accepted 10 January 2014; published online 27 January 2014)

\begin{abstract}
The optical properties of metallic spherical nanoparticles embedded in host liquid matrix are studied. Extended Maxwell-Garnett-Mie formulation which accounts for size dispersion, the intrinsic confinement, and extrinsic size effect, is proposed for the calculation of the effective dielectric function and absorption coefficient of size dispersion of colloidal solution of Au and Ag nanoparticles in water. We demonstrate that the size distribution induces an inhomogeneous broadening and an increase of the amplitude of the plasmon band. A large redshift of the plasmon band is also observed for silver nanoparticles. Compared to the conventional Maxwell Garnett theory, we demonstrated that this model gives better description of the measured absorption spectra of colloidal gold solutions. (C) 2014 AIP Publishing LLC. [http://dx.doi.org/10.1063/1.4862995]
\end{abstract}

\section{INTRODUCTION}

Noble metal nanoparticles (NPs) are increasingly utilized in many fields of applied sciences, such as sensors, ${ }^{1}$ optical filtering, ${ }^{2,3}$ optoelectronics devices, ${ }^{4}$ information storage, ${ }^{5}$ and nonlinear optics. ${ }^{6}$ Indeed, these NPs exhibit unique optical properties such as strong surface plasmon resonances (SPR) coming from the collective oscillations of their conduction electrons. The characteristics of the SPR depend on the dielectric constant of the host medium and on size and shape of the NPs. ${ }^{7,8}$ In the case of spherical NPs, the SPR diameter dependence is related to both intrinsic confinement and extrinsic dynamic effects. ${ }^{9,10}$ The intrinsic confinement traduces the modification of the dielectric function of metal due to the finite size of the NPs while the extrinsic dynamic effect comes from the non-uniformity of the electric field inside the NPs.

Conventional preparation routes unavoidably conduct to NPs showing a size distribution which induces drastic changes in the optical properties of the effective composite medium which consists of NPs embedded in a host matrix. Indeed, as experimentally revealed by Billaud et al.,${ }^{11}$ the size distribution of NPs generates an inhomogeneous broadening of the SPR band. Amendola and Meneghetti ${ }^{12}$ introduced the size distribution in the Mie Gans theory by weighting the extinction cross section of individual nanoparticles by the size distribution. However, Mie theory does not consider interaction between nanoparticles, so this approach is only restricted to dilute nanoparticle arrays. Moreover, the complex effective dielectric function of the composite medium, which is a key parameter for ellipsometric investigation, cannot be directly extracted from the Mie theory. Ellipsometric measurements performed on silver NPs with highly dispersed size revealed a broad SPR linewidth ${ }^{13}$ which could not have been interpreted

\footnotetext{
a) Author to whom correspondence should be addressed. Electronic mail: yann.battie@univ-lorraine.fr
}

in terms of conventional effective medium theories such as Maxwell Garnett (MG) theories. ${ }^{14}$ Indeed, MG theory is extensively used to describe the optical properties of collections of metallic nanoparticules embedded in a host medium. However, this theory, which originally derives by invoking the dipolar character of the NPs, takes into account their mean size and neglects their size distribution. Few approaches were proposed to introduce the size dispersion in effective medium theory. ${ }^{15-18}$ Barrera et al. ${ }^{15}$ extended the effective medium theory to polydisperse NPs by taking into account the correlated fluctuation among the induced dipole moment and internal field. However, retardation effects, which are predominant for NPs radius higher than $10 \mathrm{~nm},{ }^{10,19}$ were neglected by these authors. Retardation effects induce a redshift of the plasmon resonance as the nanoparticle radius increases. ${ }^{20} \mathrm{On}$ the other hand, Chylek and Srivastava ${ }^{16}$ developed an effective medium model which consisted in annulling the forwardscattering amplitude of a cell of the composite medium, immersed inside the effective homogeneous background. The size distribution was included through the dipolar magnetic contribution of the NPs. This model did not take into account intrinsic confinement effects observed for NPs size smaller than the mean free path of the conduction electrons. Moreover, as shown by Ruppin, ${ }^{21}$ the magnetic dipolar contribution can be neglected for noble metallic NPs. A realistic extension of the Maxwell Garnett model was reported by Banyai and $\operatorname{Koch}^{18}$ and successfully applied to determine the optical properties of silicon quantum dots embedded in a dielectric matrix. $^{22}$ However, this model was based on the numerical calculation of the average polarizability of the ensemble of NPs in quasistatic approximation. In other words, it did not take into account dynamic effects such as retardation effects that occur for large NP diameter.

In this paper, a modified effective medium theory based on dipolar approximation is developed to take into account the size distribution of spherical NPs, the intrinsic confinement effects and extrinsic dynamic effects. Our approach is 
similar to the reported one by Niu et $a .^{23}$ to describe the optical properties of a mixture of nanoparticles distributed in shape, by averaging the dipole moments of individual nanoparticles. The averaging was performed by weighting dipole moments over the relative abundance of each particle. $^{23}$ The influence of the diameter dispersion on the plasmon resonance of colloidal gold and silver NPs dispersed in water is investigated through several simulations. The theoretical model can be validated by comparing these simulations to experimental absorption spectra of colloidal gold solutions. We demonstrate that the effective optical properties of the composite medium depend on both the size distribution and the nature of the NPs.

\section{THEORY}

\section{A. Maxwell-Garnett (MG) and Maxwell-Garnett Mie theories (MGM)}

In dipolar approximation, the effective dielectric function $\varepsilon_{\text {eff }}$ of the composite medium which consists of spherical inclusions embedded in a host matrix is related to the polarizability $\alpha$ of the inclusions through the Clausius-Mossotti relation: ${ }^{9,10}$

$$
\frac{\varepsilon_{e f f}-\varepsilon_{m}}{\varepsilon_{e f f}+2 \varepsilon_{m}}=\frac{f}{\bar{R}^{3}} \alpha(\bar{R})
$$

where $\varepsilon_{\mathrm{m}}$ is the dielectric function of the matrixand $\bar{R}$ and $f$ are the mean radius of NPs radius and the volume fraction of the inclusions, respectively. Note that the size of the inclusion must be smaller than the wavelength and the volume fraction must be small enough to satisfy the dipolar approximation. In quasistatic limit, the polarizability of an inclusion with a radius $\mathrm{R}$ is given by

$$
\alpha_{M G}(R)=R^{3} \frac{\varepsilon_{n p}-\varepsilon_{m}}{\varepsilon_{n p}+2 \varepsilon_{m}},
$$

where $\varepsilon_{\mathrm{np}}$ is the dielectric function of the inclusion, i.e., the NPs. When substituted into Eq. (1), this yields the usual MG formula. ${ }^{14}$ The radius does not explicitly appear in the MG formula, and only intrinsic confinement is taken into account. Indeed, quasistatic approximation is only valid for homogeneous field inside the NPs. As a consequence, the polarizability must be redefined to incorporate dynamic effects in effective medium theory. Doyle ${ }^{24}$ suggested that a size-dependent extension of the MG formula can be obtained by deriving the electric dipole polarizability from the Mie theory:

$$
\alpha_{M G M}(R)=\frac{3 i \lambda^{3}}{16 \pi^{3} \varepsilon_{m}^{3 / 2}} a_{1}(R),
$$

where $a_{1}$ is the first electric Mie coefficient given by

$$
a_{1}(R)=\frac{\sqrt{\varepsilon_{n p}} \Psi_{1}\left(2 \pi R \sqrt{\varepsilon_{n p}} / \lambda\right) \Psi_{1}^{\prime}\left(2 \pi R \sqrt{\varepsilon_{m}} / \lambda\right)-\sqrt{\varepsilon_{m}} \Psi_{1}\left(2 \pi R \sqrt{\varepsilon_{m}} / \lambda\right) \Psi_{1}^{\prime}\left(2 \pi R \sqrt{\varepsilon_{n p}} / \lambda\right)}{\sqrt{\varepsilon_{n p}} \Psi_{1}\left(2 \pi R \sqrt{\varepsilon_{n p}} / \lambda\right) \xi_{1}^{\prime}\left(2 \pi R \sqrt{\varepsilon_{m}} / \lambda\right)-\sqrt{\varepsilon_{m}} \Psi_{1}\left(2 \pi R \sqrt{\varepsilon_{m}} / \lambda\right) \xi_{1}^{\prime}\left(2 \pi R \sqrt{\varepsilon_{n p}} / \lambda\right)},
$$

where $\Psi_{1}$ and $\xi_{1}$ are the first order Riccati Bessel functions. Note that for small NP size, Eqs. (2) and (3) are equivalent. The modified long wavelength approximation ${ }^{7}$ which modifies the quasistatic polarizability to introduce radiative damping and dynamic depolarization is only a four order expansion of Eq. (3). In the considered radius range, i.e., for radius smaller than $25 \mathrm{~nm}$, MLWA and Eq. (3) are similar. The combination of Eqs. (1), (3), and (4) gives the so-called Maxwell-Garnett Mie theory (MGM) and allows calculating the effective dielectric function by considering both intrinsic confinement and extrinsic dynamic effects. ${ }^{9}$

Although conventional Mie theory well describes light absorption in dilute dispersions of weakly interacting spheres, this single-particle approach fails to interpret the interactions in nanoparticle arrays. ${ }^{25}$ On the other hand, MGM takes into account dipolar-interactions between nanoparticles. In other words, contrary to the Mie theory, MGM is more adequate to describe the optical properties of a collection of small nanoparticles. In addition, contrary to the Mie theory, MGM determines the effective dielectric function of nanoparticle arrays which is a useful parameter for ellipsometric, reflectometric, or interferometric measurements.

\section{B. Modified Maxwell-Garnett Mie theory (MMGM)}

Realistic composite materials are made of NPs with variable sizes. As a consequence, each NP exhibits its own polarizability value. Considering the mean field theory, the Eqs. (1) and (3) should be revised by taking into account the mean polarizability of the NPs. This gives the Modified Maxwell-Garnett Mie theory (MMGM):

$$
\frac{\varepsilon_{e f f}-\varepsilon_{m}}{\varepsilon_{e f f}+2 \varepsilon_{m}}=\frac{3 i \lambda^{3}}{16 \pi^{3} \varepsilon_{m}^{3 / 2}} \frac{f}{\bar{R}^{3}} \int_{R_{\min }}^{R_{\max }} P(R) a_{1}(R) d R
$$

where $P(R)$ is the NPs size distribution. $R_{\min }$ and $R_{\max }$ represent the lower and upper bounds of the size distribution. The constant average nanoparticles radius $(\bar{R})$, which appears in the denominator of Eq. (5), comes from the volume fraction introduced in the Clausius Mossoti relation (1). Note that the MMGM respects the Kramers-Kronig relations and is valid for any distribution law $\mathrm{P}(\mathrm{R})$.

As pointed out by Ruppin, ${ }^{21}$ the effective dielectric function has a physical meaning only if the scattering cross section of the NPs is smaller than their absorption cross section. This limits the validity of the effective medium theory, and thus, 
the MMGM theory to radius less than $25 \mathrm{~nm}$ for gold and silver NPs. ${ }^{10}$

\section{Dielectric function of metallic nanoparticles}

The dielectric function of noble metal NPs such as silver and gold consists of two contributions accounting for interband and intraband electron transitions that involve bound and free electrons, respectively. In classical approach, confinement effects appear when the mean free path of conduction electron is limited by the nanoparticle size. Assuming that only the free electrons are affected by the NPs size,${ }^{26}$ the dielectric function of metallic NPs can be expressed in the following way: ${ }^{10}$

$$
\varepsilon_{n p}(R)=\varepsilon_{\text {bulk }}-\frac{\omega_{p}^{2}}{\omega\left(\omega+i \Gamma_{0}\right)}+\frac{\omega_{p}^{2}}{\omega\left(\omega+i\left(\Gamma_{0}+A \frac{v_{f}}{R}\right)\right)},
$$

where $\varepsilon_{\text {bulk }}, \omega_{\mathrm{p}}, v_{f}$, and $\Gamma_{0}$ are the tabulated dielectric function of the bulk, ${ }^{27,28}$ the plasma frequency, the Fermi velocity of free electrons, and electron damping, respectively. A is a constant which depends on the details of the electron-nanoparticle surface scattering process. For spherical nanoparticles, classical approach gives $\mathrm{A}=1$ or 0.75 for isotropic and diffusive electron scattering, respectively ${ }^{29}$ while quantum theory yield to 0.75 or 0.86 values. Due to chemical interface damping, this constant also depends on the surrounding medium. ${ }^{30}$ As example, measurements performed on spherical silver/silica core shell nanoparticles yield to $\mathrm{A}=0.6$, i.e., a slightly smaller value than the predicted one by classical and quantum theory. ${ }^{31,32}$ In this paper, the constant $\mathrm{A}$ is assumed to be equal to one. Note that an overestimation of A could induce a broadening of the SPR band. As shown by the density functional theory, ${ }^{26}$ this dispersion law can be used for NP radius above $1 \mathrm{~nm}$. In other words, the combination of Eqs. (5) and (6) can be used for spher- ical nanoparticles with radius ranging from $1 \mathrm{~nm}$ to $25 \mathrm{~nm}$ The first two terms of Eq. (6) traduce the interband dielectric function while the third term describes the intraband dielectric function. This latter term contains intrinsic confinement effects due to the limitation of the mean free path of free electrons by the scattering from the particle surface. For silver NPs, $\varepsilon_{\text {bulk }}$ was taken from Palik's handbook ${ }^{27}$ and parameters $\omega_{p}, \Gamma_{0}$ and $v_{f}$ were fixed to $8.4 \mathrm{eV}, 0.08 \mathrm{eV}$, and 1.39 $\times 10^{6} \mathrm{~m} / \mathrm{s}$, respectively. For gold NPs, $\varepsilon_{\text {bulk }}$ was extracted from the Ref. 28 while parameters $\omega_{p}, \Gamma_{0}$ and $v_{f}$ were $9.06 \mathrm{eV}, 0.077 \mathrm{eV}$, and $1.4 \times 10^{6} \mathrm{~m} / \mathrm{s}$, respectively.

\section{SIMULATIONS}

\section{A. Size effects}

The effective complex dielectric function of metallic spherical NPs embedded in host liquid matrix was calculated for different NPs sizes. The simulations of the optical response of aqueous colloidal solutions of $\mathrm{Au}$ and $\mathrm{Ag}$ were performed using MG and MGM without considering the size dispersion. The mean radii of NPs were $1,2,5,10$, and $20 \mathrm{~nm}$ and the volume fraction was $1 \%$. The results are given in Fig. 1 and Fig. 2 for $\mathrm{Au}$ and Ag, respectively.

The spectra of real and imaginary parts of the effective dielectric function calculated by Maxwell-Garnett model show a slight shift of the plasmon resonance toward higher energies with increasing size accompanied by a narrowing and an increase of the amplitude of the plasmon band. The evolution of the full width at half maximum (FWHM) of the plasmon band can be attributed to the intrinsic confinement effects. ${ }^{10}$ Indeed, the damping coefficient of free electrons, which is inversely proportional to the NP radius, may induce broadening of the plasmon band for small sizes. The amplitudes increase of the dielectric function with increasing NPs size is much more important for $\mathrm{Ag}$ compared to Au NPs. In the case of

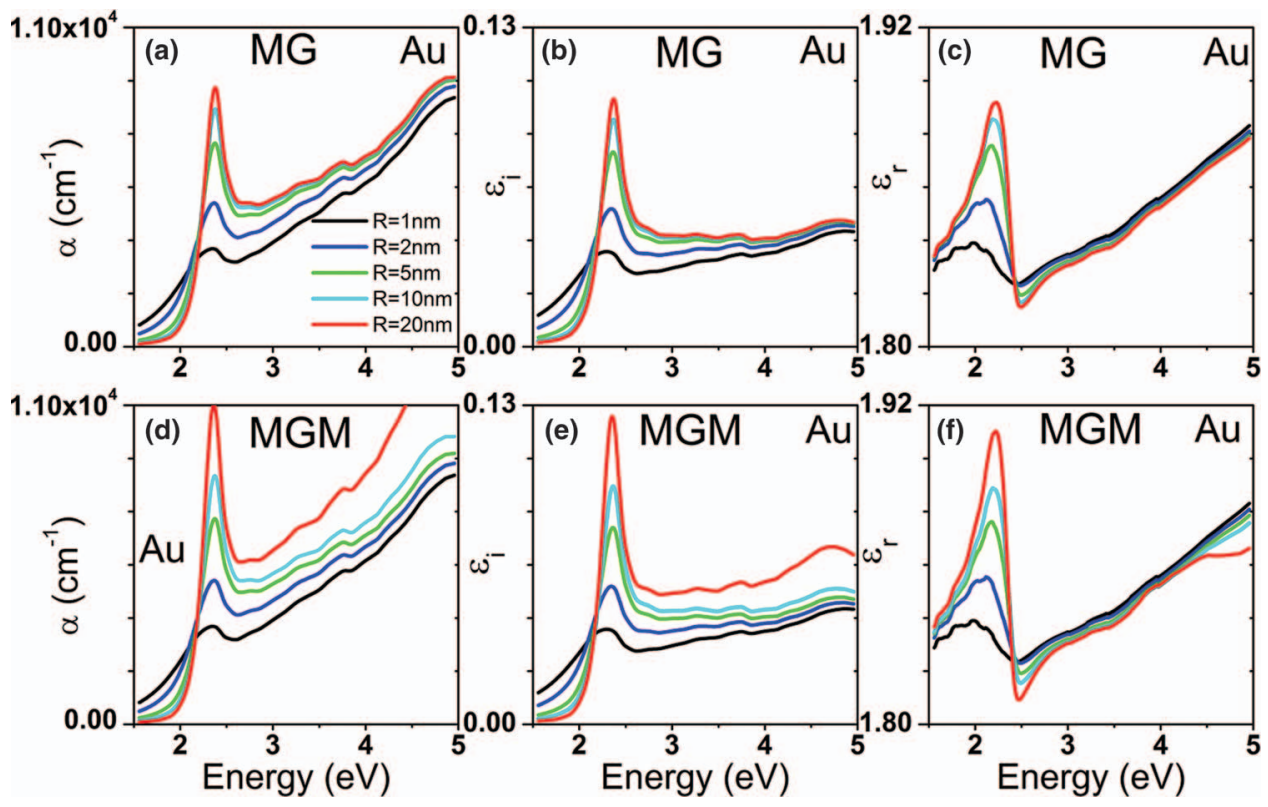

FIG. 1. [(a), (d)] Absorption coefficient and [(b), (c), (e), (f)] effective complex dielectric function of monodisperse gold NPs in water calculated from MG and MGM, respectively. The volume fraction is fixed at $1 \%$. 



FIG. 2. [(a), (d)] Absorption coefficient and [(b), (c), (e), (f)] effective complex dielectric function of monodisperse silver NPs in water calculated from MG and MGM, respectively. The volume fraction is fixed at $1 \%$.

$\mathrm{Au}$ NPs, interband transition threshold is closed to the plasmon resonance. As a consequence, the interband transitions of $\mathrm{Au}$ NPs reduce the amplitude of the plasmon band ${ }^{33}$ by $25 \%$ with respect to that of silver NPs. Therefore, the difference between dielectric functions calculated by MG and MGM models is much more marked for silver NPs. This difference is caused by the dynamic depolarization effects which are weakly visible in gold due to the presence of interband transitions closed to the plasmon energy. ${ }^{10}$ In the UV spectral range, the effective dielectric function, calculated according to the MG model weakly depends on NP size. In this model, the NP radius is only contained in the Drude part (Eq. (6)), i.e., the plasmon resonance. By contrast, the dielectric function is strongly related to the radius in MGM theory. This radius dependence introduces a variation of dielectric function amplitudes beyond the plasmon resonance at around $2.8 \mathrm{eV}$ for $\mathrm{Au}$ and $3.2 \mathrm{eV}$ for $\mathrm{Ag}$.

In agreement with Kreibig and Vollmer, ${ }^{10}$ the red shift observed in the MGM model is attributed to dynamic depolarization effects. This phenomenon can be explained by the non-instantaneous uniformity of the electric field inside the NP. ${ }^{10}$ By performing a four order expansion of the first Mie coefficient, Kuwata et al. ${ }^{20}$ have shown that dynamic depolarization can be decomposed into two effects: retardation effect and radiation loss effect. Retardation effect which brings in the second order term of the expansion of the first electric Mie coefficient induces a redshift of the plasmon resonance. In the other hand, radiation loss effects which appears in the third order term of the expansion of the first electric Mie coefficient introduces a broadening of the plasmon resonance as the nanoparticle radius increases. In agreement with Kreibig and Vollmer, ${ }^{10}$ radiation loss effect is negligible in the $1 \mathrm{~nm}-$ $25 \mathrm{~nm}$ radius range and only retardation effect are observed for nanoparticle radius larger than $10 \mathrm{~nm}$ (Figs. 1-3).
In order to clearly show the difference between MG and MGM models, the evolution of FWHM of the plasmon band has been plotted against resonance energy for both $\mathrm{Au}$ (Fig. 3(a)) and Ag (Fig. 3(b)) for NPs radii ranging from 1 to $25 \mathrm{~nm}$.

For small particles size $(<3 \mathrm{~nm}$ for $\mathrm{Ag}$ and $<6 \mathrm{~nm}$ for $\mathrm{Au}), \mathrm{MGM}$ and $\mathrm{MG}$ give same results. Indeed, the resonance energy exhibits a blue shift with increasing NPs radius. This blue shift is attributed to intrinsic confinement effect. ${ }^{10}$ The difference between MGM and MG becomes significant from $3 \mathrm{~nm}$ for Ag NPs and $6 \mathrm{~nm}$ for Au NPs. With increasing radius, this difference is more pronounced for $\mathrm{Ag}$ for which the resonance exhibits a strong red shift when calculated by MGM theory. This difference between silver and gold is due to the prominent inter-band transitions of gold that minimize NP size effects. ${ }^{10}$

MG model can be applied to study only the optical responses of smaller sizes (less than $3 \mathrm{~nm}$ for $\mathrm{Ag}$ and $6 \mathrm{~nm}$ for $\mathrm{Au}$ ). However, the MGM theory can be considered as a more general model, it can describe the optical properties of both small NPs as well as the large size $(<25 \mathrm{~nm}) .^{21}$
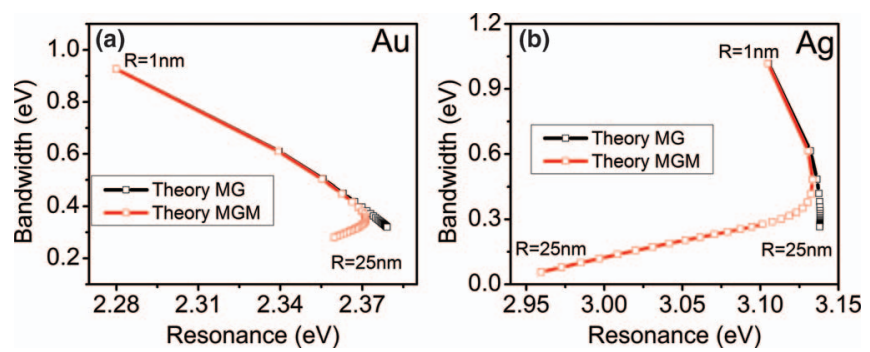

FIG. 3. Bandwidth of the plasmon band versus the resonance energy of (a) gold and (b) silver NPs in water calculated for different radius from MG and MGM. The volume fraction is fixed at $1 \%$. 


\section{B. Size dispersion effects}

Figure 4 shows the imaginary part of effective dielectric function of $\mathrm{Au}$ and $\mathrm{Ag}$ NPs calculated by taking into account size dispersion of NPs in water matrix. The simulations were performed by MMGM formulas (Eq. (5)) for a lognormal size distribution with $\sigma=1.20$ corresponding to typical distributions frequently observed in TEM histograms. ${ }^{34}$ Three different mean radii, i.e., 2, 5, and $10 \mathrm{~nm}$ were taken into consideration in calculation. A monodisperse and a normal distribution of nanoparticles are also considered. The standard deviations and the mean value of the normal distributions are deduced from the second and the first order moment of the corresponding lognormal distributions.

Simulations performed from lognormal and normal distributions give similar results. Indeed, for each mean radius, both distributions considered in Figure 4 have the same first and second central moment. Now, we focus our discussion on simulations performed from lognormal distribution. If the size distribution does not change drastically the behaviours of dielectric functions, a significant increase of both amplitude and width is however observed as the size dispersion rises. For example, for an average radius of $2 \mathrm{~nm}$ of Au NPs, the real and imaginary of the dielectric function at resonance changes from 1.83 to 1.85 and 0.056 to 0.068 , respectively. In other words, an augmentation of the real and imaginary parts of dielectric function is observed when the size distribution is introduced in MGM model.
For Ag NPs, the imaginary part of the dielectric function of $2 \mathrm{~nm}$ average size at plasmon resonance changes from 0.171 to 0.212 while its real part at resonance remains almost constant. In addition to the change of amplitude and width, a redshift is also observed for Ag NPs.

As a first approximation, by considering quasistatic polarizability without including intrinsic size effects, the change of amplitudes of optical responses can be explained by an artificial increase of the volume fraction $f_{\text {eff }}$ of NPs when the size distribution is taken into account in the model. This new effective volume fraction $\left(f_{\text {eff }}\right)$ can be written

$$
f_{\text {eff }}=\frac{f}{\bar{R}^{3}} \int_{R_{\min }}^{R_{\max }} P(R) R^{3} d R,
$$

where $f$ is the initial volume fraction value of NPs in water.

It is also interesting to consider the optical properties of these NPs obtained with MMGM formula for various values (from 1.05 to 1.80) of the size dispersion $\sigma$ for a fixed mean radius of $2 \mathrm{~nm}, 5 \mathrm{~nm}$, and $10 \mathrm{~nm}$. Figure 5 plots the evolution of the real and imaginary parts of the dielectric functions and the absorption coefficient of Au NPs with 2, 5, and $10 \mathrm{~nm}$ mean radii and for variable size dispersion $\sigma$. These results were determined from the numerical simulation of MMGM (5).

First, it is observed that both amplitude and width of the plasmon band increase with increasing $\sigma$. This result traduces an inhomogeneous broadening of the plasmon resonance. For
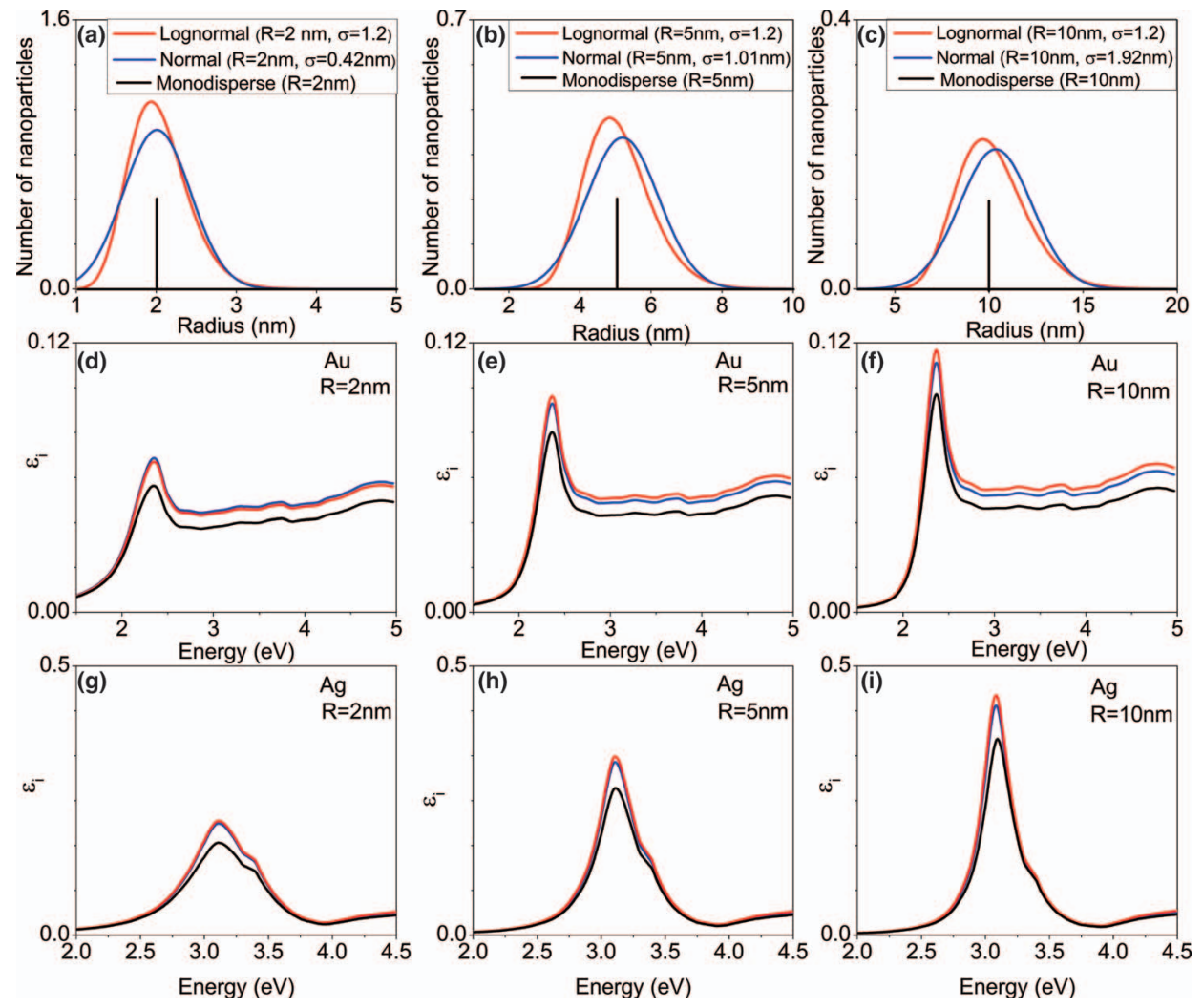

FIG. 4. [(a), b, (c)] Nanoparticles distribution used to simulate imaginary part of the complex dielectric function of [(d), (e), (f)] Au and [(g), (h), (i)] Ag NPs. Three distribution laws are investigated: a monodisperse distribution (black line), a lognormal distribution (red line), and a normal (blue line) distribution. The mean radii of the lognormal distribution are [(a), (d)] $2 \mathrm{~nm}$, [(b), (e)] $5 \mathrm{~nm}$, and [(c), (f)] $10 \mathrm{~nm}$. A 1.2 standard deviation is considered for lognormal distribution. The mean value and standard deviation of normal distributions are calculated from the first and second central moment of lognormal distributions, respectively. 



FIG. 5. Influence of the standard deviation on [(a), (b), (c)] the absorption coefficient and [(d), (e), (f), (g), (h), (i)] the complex dielectric function of gold NPs in water matrix calculated from MMGM. The mean diameters are $2 \mathrm{~nm}, 5 \mathrm{~nm}$, and $10 \mathrm{~nm}$.

Au NPs with means radius of $2 \mathrm{~nm}$, the amplitude of the imaginary dielectric function varies from 0.059 to 0.4268 as $\sigma$ varies from 1.10 to 1.80 . The same trend is observed for a mean radius of $5 \mathrm{~nm}$. In contrast, for a $10 \mathrm{~nm}$ mean radius, the amplitude of the plasmon band of Au NPs does not increase monotonously with $\sigma$. In other words, the amplitude becomes slightly constant for $\sigma$ higher than 1.6. This can be explained by an augmentation of the effective volume fraction as the $\sigma$ increases. The evolution of the effective volume fraction of Au NPs as a function of size dispersion is illustrated in Fig. 6(a) for the three mean radii.

The calculated effective volume fractions are very closes for the distributions centered at 2 and $5 \mathrm{~nm}$. In these cases, the values of $f_{\text {eff }}$ increase monotonically with $\sigma$. For a mean radius of $10 \mathrm{~nm}, f_{\text {eff }}$ increases at first monotonically up to $\sigma$ $=1.6$ and saturates above this value. This latter result is in good agreement with the fact that the dielectric functions calculated for $\sigma=1.60$ and 1.80 give similar results (Fig. 5(c)). In addition, the evolution of the plasmon resonance vs radius (Fig. 6(b)) shows that the smaller of slope variation around the radius of 2, 5, and $10 \mathrm{~nm}$ can explain the lower redshift of plasmon resonance of Au NPs.

Concerning the Ag NPs, the results are given in Fig. 7. The amplitude and width of optical responses are also strongly correlated to $\sigma$. For example, the dispersion with mean radius of $2 \mathrm{~nm}$, the amplitude of the imaginary part of dielectric function at plasmon resonance varies from 0.182 to 1.421 when $\sigma$ is increased from 1.10 to 1.80 . Unlike of $\mathrm{Au}$
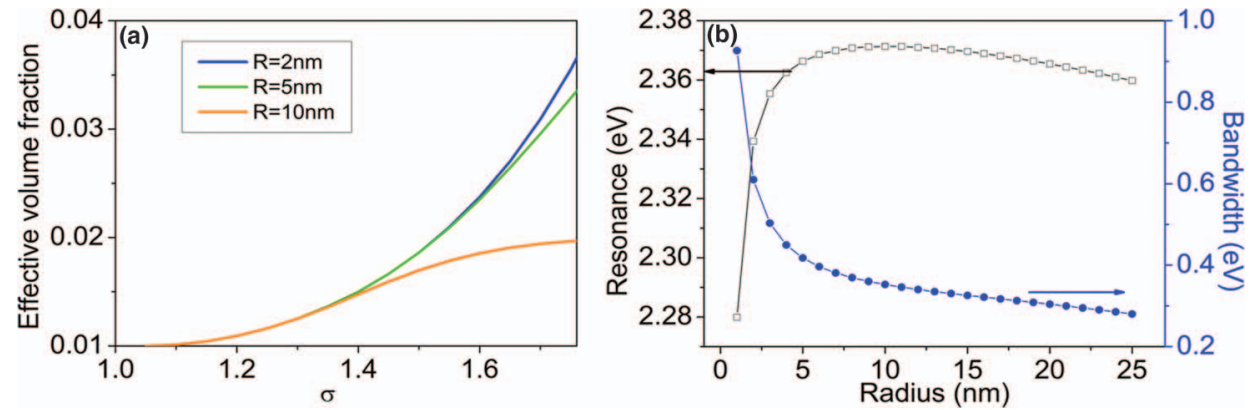

FIG. 6. (a) Evolution of the effective volume fraction with the standard deviation. (b) Resonance energy and bandwidth of the surface plasmon band of monodispersed gold NPs versus their radius. 

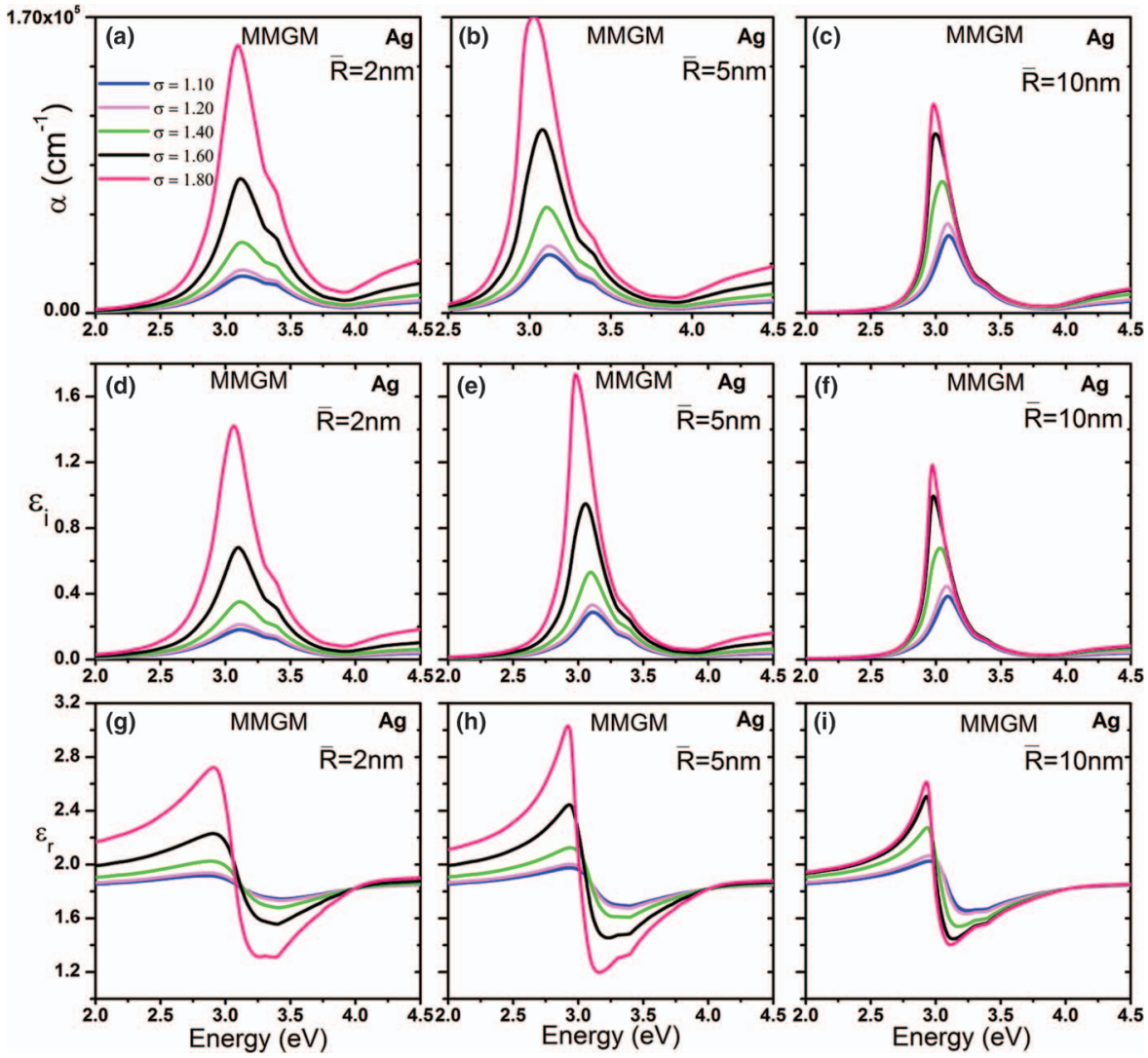

FIG. 7. Influence of the standard deviation on [(a), (b), (c)] the absorption coefficient and [(d), (e), (f), (g), (h), (i)] the complex dielectric function of silver NPs in water matrix calculated from MMGM. The mean diameters are $2 \mathrm{~nm}, 5 \mathrm{~nm}$, and $10 \mathrm{~nm}$.

NPs, a redshift of plasmon resonance is observed with the increase of $\sigma$. This is attributed to the higher size dependence of dielectric function of Ag NPs (Fig. 3(b)).

For a collection of monodisperse Ag NPs, the curve of plasmon resonance as a function of radius shows that the slope increases with the redshift (Fig. 8). Compared to Au NPs, the optical response of Ag NPs is more pronounced at high NPs size and induces a shift to lower energies. So the asymmetrical redshift is obtained by varying the size dispersion. Indeed, the redshift is more important for distribution centered at $10 \mathrm{~nm}$ than that centered at $2 \mathrm{~nm}$.

We conclude that in both cases, the size distribution dependence of the dielectric function of Au and Ag NPs has as effect an augmentation of the amplitude and an inhomo-

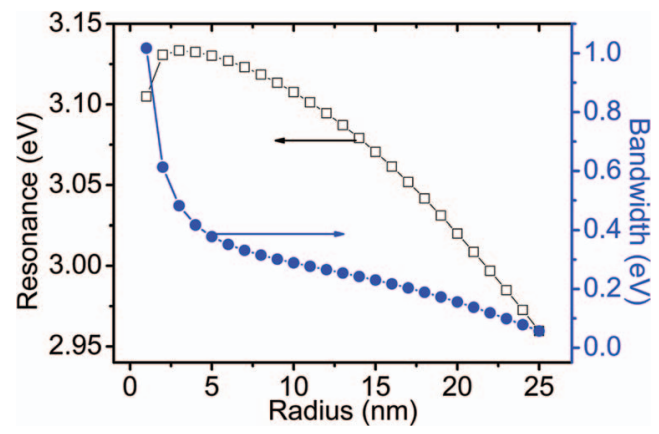

FIG. 8. Resonance energy and bandwidth of the surface plasmon band of monodispersed silver NPs versus their radius. geneous broadening of the plasmon band. Although effective volume fraction approximation (Eq. (7)) can be applied to Au, it is not valid for the Ag NPs. The main difference between these nanometals is derived from interband transitions that influence strongly the optical responses in larger optical spectrum range.
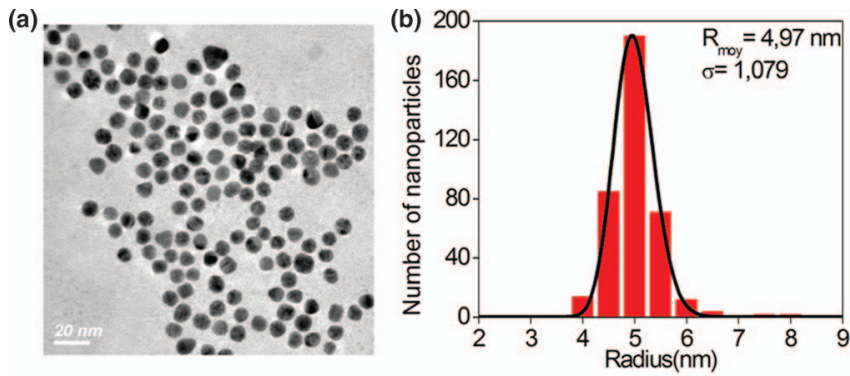

(c)
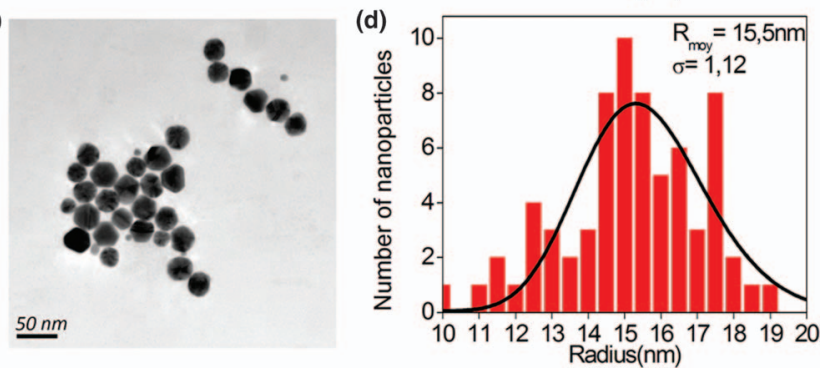

FIG. 9. [(a), (c)] TEM images and [(b), (d)] radius distribution of [(a), (b)] $\mathrm{S} 1$ and $[(\mathrm{c}),(\mathrm{d})] \mathrm{S} 2$. 

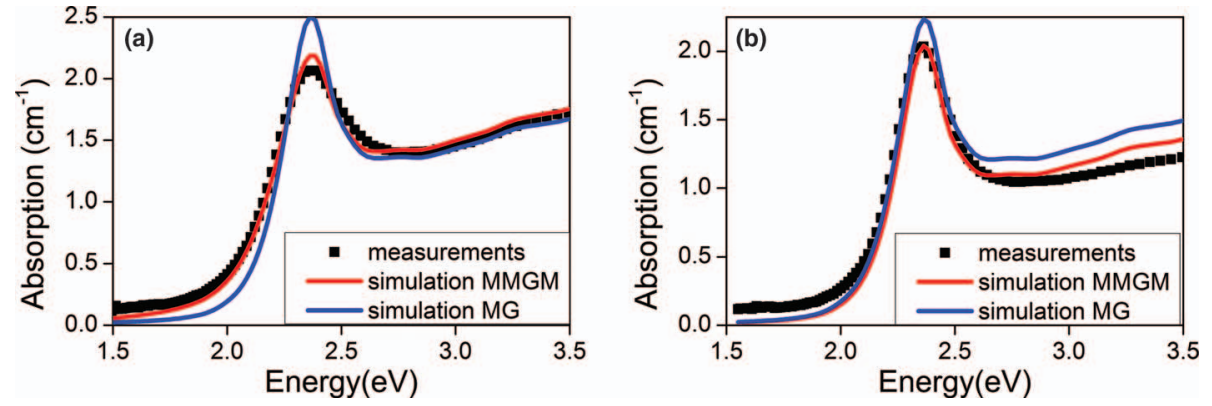

FIG. 10. Comparison between experimental absorption spectra of (a) S1 and (b) S2 and the theoretical ones by MG and MMGM.

\section{EXPERIMENTAL}

To validate the MMGM model, simulations were compared to the absorption coefficient of two commercial gold colloids $\left(S_{1}\right.$ and $\left.S_{2}\right)$ dispersed in water purchased from Sigma Aldrich. Their volume fractions, given by the supplier, are in the $1.10^{-4} \%-6.10^{-4} \%$ range. Note that measurements on $\mathrm{Ag}$ colloids are in progress and will be reported in a later paper. Transmission electron microscopy (TEM) and absorption spectroscopy were performed with a Phillips CM200 TEM, and a Horiba Jobin Yvon spectrometer. Figure 9 illustrates the TEM images of $S_{1}$ and $S_{2}$. The corresponding diameter distributions extracted from the statistical analysis of TEM images are also reported.

Although $S_{2}$ NPs seem more faceted, $S_{1}$ and $S_{2}$ can be considered as spherical. The radius distribution can be described by a lognormal distribution law. The average radius of $S_{1}$ and $S_{2}$ NPs are estimated to $5 \mathrm{~nm}$ and $15.5 \mathrm{~nm}$, respectively. The standard deviations of the $S_{1}$ and $S_{2}$ distributions are 1.08 and 1.12 , respectively.

Figure 10 compares the measured absorption coefficient of $S_{1}$ and $S_{2}$ to the simulated ones from the MMGM and the conventional MG theories. Note that the simulations were performed without free parameter excepted for the volume fraction. To respect the domain of validity of Eqs. (5) and (6), the lower and upper limits of integral in Eq. (5) are $1 \mathrm{~nm}$ and $25 \mathrm{~nm}$, respectively. The latter, estimated from the MMGM theory, are $1.9 \times 10^{-4} \%$ and $2.6 \times 10^{-4} \%$, for $S_{1}$ and $S_{2}$, respectively. This is in good agreement with the volume fraction range given by the supplier. As shown in Figure 10, both models precisely predict the SPR energy. However, the simulated spectra obtained from the MMGM theory is in much better agreement with the measured spectra than that obtained from the MG theory. The small deviations, mainly observed for $\mathrm{S}_{2}$, can be reasonably attributed to the facetted shape of $\mathrm{S}_{2}$ NPs. In particular, the MMGM theory allows predicting the energy, amplitudes, and width of the SPR band. These results also suggest that the assumption that the scattering event constant $\mathrm{A}$ is close to 1 is valid. This unambiguously demonstrates that the NP size distribution must be considered in order to describe the optical properties of a collection of NPs with a high accuracy.

\section{CONCLUSIONS}

In conclusion, a new model based on dipolar approximation was developed to describe the effective optical properties of a collection of spherical NPs by taking into account the NP size distribution, the intrinsic confinement, and the extrinsic dynamic effects. The complex effective dielectric function and the absorption coefficient of gold and silver NPs dispersed in water were investigated for different lognormal size distributions. The size distribution was found to induce an inhomogeneous broadening and an increase of the amplitude of the SPR band. A large redshift of the SPR was also observed for silver NPs. Compared to the conventional MG theory, the MMMG model provides a better description of the absorption spectra of colloidal gold solutions. Thus, this model is a new step forwards the comprehension of the optical properties of composite materials.

${ }^{1}$ J. N. Anker, W. Paige Hall, O. Lyandres, N. C. Shah, J. Zhao, and R. P. Van Duyne, Nat. Mater. 7, 442 (2008).

${ }^{2}$ G. Carotenuto, Appl. Organomet. Chem. 15, 344 (2001).

${ }^{3}$ R. Sayah, L. Nadar, F. Vocanson, Y. Battie, S. Reynaud, R. Vera, A. Boukenter, and N. Destouches, Microporous Mesoporous Mater. 139, 45 (2011).

${ }^{4}$ Y. Dirix, C. Bastiaansen, W. Caseri, and P. Smith, Adv. Mater. 11, 223 (1999).

${ }^{5}$ T. Fournel, N. Destouches, Y. Battie, L. Bois, and Y. Boutant, J. Phys.: Conf. Ser. 139, 012019 (2008).

${ }^{6}$ S. Porel, S. Singh, S. S. Harsha, D. N. Rao, and T. P. Radhakrishnan, Chem. Mater. 17, 9 (2005).

${ }^{7}$ K. L. Kelly, E. Coronado, L. L. Zhao, and G. C. Schatz, J. Phys. Chem. B 107, 668 (2003).

${ }^{8}$ C. Noguez, J. Phys. Chem. C 111, 3806 (2007).

${ }^{9}$ V. Myroshnychenko, J. Rodriguez-Fernandez, I. Pastoriza-Santos, A. M. Funston, C. Novo, P. Mulvaney, L. M. Liz-Marzan, and F. J. Garcia de Abajo, R. Soc. Rev. 37, 1792 (2008).

${ }^{10} \mathrm{U}$. Kreibig and M. Vollmer, Optical Properties of Metal Clusters (Springer, Berlin, 1995).

${ }^{11}$ P. Billaud, J.-R. Huntzinger, E. Cottancin, J. Lermé, M. Pellarin, L. Arnaud, M. Broyer, N. Del Fatti, and F. Vallée, Eur. Phys. J. D 43, 271 (2007).

${ }^{12}$ V. Amendola and M. Meneghetti, J. Phys. Chem. C 113, 4277 (2009).

${ }^{13}$ Y. Battie, N. Destouches, F. Chassagneux, D. Jamon, L. Bois, N. Moncoffre, and N. Toulhoat, Opt. Mater. Express 1, 1019 (2011).

${ }^{14}$ G. J. C. Maxwell Garnett, Philos. Trans. R. Soc. London, Ser. A 203, 385 (1904).

${ }^{15}$ R. G. Barrera, P. Villasenir-Gonzalez, W. L. Mochan, and G. Monsivais, Phys. Rev. B 41, 7370 (1990).

${ }^{16}$ P. Chýlek and V. Srivastava, Phys. Rev. B 27, 5098 (1983).

${ }^{17}$ L. G. Grechko, V. N. Pustovit, and K. W. Whites, Appl. Phys. Lett. 76, 1854 (2000)

${ }^{18}$ L. Banyai and S. X. Koch, Semiconductor Quantum Dots (World Scientific, Singapore, 1993).

${ }^{19}$ S. Berciaud, L. Cognet, P. Tamarat, and B. Lounis, Nano Lett. 5, 515 (2005).

${ }^{20}$ H. Kuwata, H. Tamaru, K. Esumi, and K. Miyano, Appl. Phys. Lett. 83, 4625 (2003)

${ }^{21}$ R. Ruppin, Opt. Commun. 182, 273 (2000).

${ }^{22}$ A.-S. Keita and A. En Naciri, Phys. Rev. B 84, 125436 (2011). 
${ }^{23}$ Z. Niu, Y.-R. Zhen, M. Gong, Q. Peng, P. Nordlander, and Y. Li, Chem. Sci. 2, 2392 (2011).

${ }^{24}$ W. T. Doyle, Phys. Rev. B 39, 9852 (1989).

${ }^{25}$ S. K. Ghosh and T. Pal, Chem. Rev. 107, 4797 (2007)

${ }^{26}$ Y. He and T. Zeng, J. Phys. Chem. C 114, 18023 (2010).

${ }^{27}$ E. D. Palik, Handbook of Optical Constants of Solids (Academic Press, Orlando, 1985).

${ }^{28}$ P. B. Johnson and R. W. Christy, Phys. Rev. B 6, 4370 (1972).

${ }^{29}$ E. A. Coronado and G. C. Schatz, J. Chem. Phys. 119, 3926 (2003).
${ }^{30}$ H. Hövel, S. Fritz, A. Hilger, U. Kreibig, and M. Volmer, Phys. Rev. B 48(24), 18178 (1993).

${ }^{31}$ H. Baida, P. Billaud, S. Marhaba, D. Christofilos, E. Cottancin, A. Crut, J. Lerme, P. Maioli, M. Pellarin, M. Broyer, N. Del Fatti, F. Vallee, A. Sanchez-Iglesias, I. Pastoriza-Santos, and L. M. Liz-Marzan, Nano Lett. 9, 3463 (2009).

${ }^{32}$ G. V. Hartland, Chem. Rev. 111, 3858 (2011).

${ }^{33}$ M. I. Stockman, Opt. Express 19, 22029 (2011).

${ }^{34}$ B. Roy and D. Chakravorty, J. Phys.: Condens. Matter 2, 9323 (1990). 